\title{
NEW CRITERIA FOR THE WEAK RADON-NIKODÝM PROPERTY RELATED TO SET-VALUED OPERATORS
}

\author{
KEUN YOUNG LEE
}

\begin{abstract}
In this paper, we deal with new equivalent conditions for the localized weak Radon-Nikodým property in dual Banach space related to set-valued operators. First, we introduce the geometric definition of the weak RadonNikodým property and the weakly fragmented set-valued operator. Next, using the weakly fragmented mapping, we reveal the relation between the weak Radon-Nikodým property and the weakly single-valued operator. Finally, using this relation and the concept of the exposed point, the main theorem is given together with some applications.
\end{abstract}

1. Introduction and the main results. Several contributions to the detection of copies of $\ell_{1}$ were made in the sixties by Pelczynski. The following theorem is given by Pelczynski (see [10]).

Theorem 1.1. Let $X$ be a separable Banach space. Then the following are equivalent.

(a) $X$ contains a copy of $\ell_{1}$.

(b) $X^{*}$ contains a copy of $\ell_{1}([0,1])$.

(c) $X^{*}$ contains a copy of $L_{1}([0,1])$.

After this theorem, many mathematicians tried to characterize $\ell_{1} \nsubseteq$ $X$ and find a nonseparable dual Banach space which contains no $\ell_{1}$. In 1973, James constructed a Banach space JT (called the James tree space) that is separable, contains no $\ell_{1}$ and has nonseparable dual (see $[\mathbf{6}, \mathbf{8}]$ ). In 1974, Rosenthal provided the true understanding of $\ell_{1}$ 's absence by proving the following theorem (called Rosenthal's $\ell_{1}$ theorem) [12].

2010 AMS Mathematics subject classification. Primary 46B22, Secondary $46 \mathrm{G} 10$.

Keywords and phrases. The weak Radon-Nikodým property, weakly fragmented set-valued operator, weakly single-valued operator, $x^{* *}$-weak* exposed point.

Received by the editors on May 20, 2010, and in revised form on September 29, 2013.

DOI: $10.1216 /$ RMJ-2015-45-5-1511

Copyright (C)2015 Rocky Mountain Mathematics Consortium 
Theorem 1.2. (Rosenthal's $\ell_{1}$ theorem). Each bounded sequence in a Banach space $X$ has a weakly Cauchy subsequence if and only if $X$ contains no isomorphic copy of $\ell_{1}$.

Shortly after Rosenthal's $\ell_{1}$ theorem, a number of classical characterizations were formulated, as follows.

Theorem 1.3. [3]. Each of the following statements characterizes Banach space $X$ that contains no copy of $\ell_{1}$.

(a) (Haydon). Each $x^{* *}$ in $X^{* *}$ is (weak ${ }^{*}$-) measurable with respect to each regular Borel probability measure on $\left(B_{X^{*}}\right.$, weak $\left.{ }^{*}\right)$.

(b) (Pelczynski). Every bounded linear $T: L_{1} \rightarrow X^{*}$ is a DunfordPettis operator.

(c) (Musial-Janika). For each finite complete measure space $(\Omega, \Sigma, \mu)$ and each $\mu$-continuous $X^{*}$-valued countably additive vector measure $\nu: \Sigma \rightarrow X^{*}$ of bounded variation, there exists a Pettis integrable function $f: \Omega \rightarrow X^{*}$ such that $\nu(E)=P-\int_{E} f d \mu$ for all $E \in \Sigma$.

(d) (Odell). Every weak ${ }^{*}$ compact convex subsets of $X^{*}$ is the normclosed convex hull of its extreme points.

A Banach space satisfying Theorem 1.3 (c) is said to have the weak Radon-Nikodým property (seen in [9]). In 1983, Saab and Saab provided the geometrical characterization of the weak Radon-Nikodým property for dual Banach spaces (see [15]). Furthermore, Riddle, Saab and Uhl introduced the localized weak Radon-Nikodým property, and they provided equivalent conditions for the localized weak RadonNikodým property in dual Banach spaces.

Our aim in this paper is to provide new equivalent conditions for the localized weak Radon-Nikodým property related to set-valued operators and perturbed lower semicontinuous functions. In Section 2, we fix notation and introduce basic properties of the set-valued operator. In Section 3, we prove basic theorems involving the notions of the weak Radon-Nikodým property, weakly fragmented mappings, and minimal weak*-compact upper semicontinuous mappings. In Section 4, we investigate the notions of weak ${ }^{*}$-minimum and $x^{* *}$-weak ${ }^{*}$-strongly exposed points where $x^{* *}$ is in $X^{* *}$ and then we establish our main theorem. 
2. Preliminaries. Let $X$ be a Banach space and $Y$ a topological space. A nonempty bounded subset $A$ of $X$ is said to be dentable if, for every $\varepsilon>0$, there exists an open half-space $V$ in $X$ such that $A \cap V \neq \emptyset$ and $\operatorname{diam}(A \cap V)<\varepsilon$, that is, for every $\varepsilon>0$, there exist $x^{*} \in X^{*}$ and $\alpha>0$ such that the slice

$$
S\left(x^{*}, A, \alpha\right)=\left\{x \in A:\left\langle x^{*}, x\right\rangle>\sup \left\langle x^{*}, A\right\rangle-\alpha\right\}
$$

has diameter less than $\varepsilon$. A subset $A$ of $X$ is said to have the RadonNikodym property if every nonempty bounded subset of $A$ is dentable. Also, a nonempty bounded subset $A$ of $X^{*}$ is said to be weak*-dentable if, for every $\varepsilon>0$, there exists a weak*-open half-space $V$ in $X^{*}$ such that $A \cap V \neq \emptyset$ and $\operatorname{diam}(A \cap V)<\varepsilon$, and a subset $A$ of $X^{*}$ is said to have the weak* Radon-Nikodým property (that is, the Radon-Nikodým property) if every nonempty bounded subset of $A$ is weak*-dentable. For more on these topics, we refer to the lecture notes by Bourgin [1] and Phelps [11].

Let $T: X \rightarrow 2^{Y}$ be a set-valued operator from $X$ into nonempty subsets of $Y$. The effective domain of $T$ is the set $\operatorname{dom}(T)=\{x \in X$ : $T(x) \neq \emptyset\}$. Then, $T$ is upper semicontinuous at $x \in X$ provided, for any open $V$ containing $T(x)$, there is an open neighborhood $U$ of $x$ such that $T(U) \subset V$. When $Y$ is a linear topological space with topology $\tau$, an upper semicontinuous with nonempty $\tau$-compact convex values is called $\tau$-cusco. The graph of $T$ is denoted by

$$
G(T)=\{(x, y) \in X \times Y: y \in T(x)\} .
$$

We partially order these set-valued operators by the inclusion ordering on their graphs. We will usually write $T_{1} \subset T_{2}$ in place of $G\left(T_{1}\right) \subset$ $G\left(T_{2}\right)$. A cusco operator is said to be minimal if it does not properly contain any other cusco operator. It is well known that a minimal $\tau$ cusco $T: Z \rightarrow 2^{Y}$ from a topological space $Z$ into a Hausdorff locally convex linear topological space $(Y, \tau)$ satisfies the following minimality properties (see, e.g., [4]).

(i) For any open set $U$ in $Z$ and any $\tau$-open half-space $V$ in $Y$ with $T(U) \cap V \neq \emptyset$ there exists a nonempty open set $U^{\prime} \subset U$ such that $T\left(U^{\prime}\right) \subset V$.

(ii) For any open set $U$ in $Z$ and any $\tau$-closed convex set $C$ in $Y$ with $T(U) \nsubseteq C$ there exists a nonempty open set $U^{\prime} \subset U$ such that $T\left(U^{\prime}\right) \cap C=\emptyset$. 
When $Y$ is a Banach space, $T$ is fragmented by norm if, for any nonempty $U$ in $X$ and $\varepsilon>0$, there is a nonempty open set $U^{\prime} \subset U$ such that norm-diam $\left(T\left(U^{\prime}\right)\right)<\varepsilon$. The following lemma provides a relation between nonempty-valued fragmented mappings and singlevalued mappings (see, e.g., [7]).

Lemma. Let $Z$ and $X$ be Banach spaces, and let $T: Z \rightarrow 2^{X}$ be a nonempty-valued mapping fragmented by norm. Then, there exists a dense $G_{\delta}$ subset $D$ of $Z$ such that, at each point of $D, T$ is single-valued and upper semicontinuous.

An extended real-valued function $f: X \rightarrow \mathbf{R} \cup\{\infty\}$ is said to be proper if its effective domain, i.e., the set $\operatorname{dom}(f):=\{x \in X: f(x)<$ $\infty\}$, is nonempty. Recall that $f$ is $\tau$-lower semicontinuous provided $\{x \in X: f(x) \leq r\}$ is $\tau$-closed in $X$ for every $r \in \mathbf{R}$. The conjugate of a function $f: X \rightarrow \mathbf{R} \cup\{\infty\}$ is the function $f^{*}$ given on $X^{*}$ by

$$
f^{*}\left(x^{*}\right):=\sup \left(x^{*}-f\right):=\sup \left\{\left\langle x^{*}, x\right\rangle-f(x): x \in X\right\} .
$$

Similarly, the conjugate of a function $f: X^{*} \rightarrow \mathbf{R} \cup\{\infty\}$ is the function $f^{*}$ given on $X$ by

$$
f^{*}(x):=\sup (x-f):=\sup \left\{\left\langle x^{*}, x\right\rangle-f\left(x^{*}\right): x^{*} \in X^{*}\right\} .
$$

The subdifferential of a convex function $f: X \rightarrow \mathbf{R} \cup\{\infty\}$ is the set-valued mapping $\partial f: X \rightarrow 2^{X^{*}}$ given by

$$
\partial f(x)=\left\{x^{*} \in X^{*}:\left\langle x^{*}, y-x\right\rangle \leq f(y)-f(x), \forall y \in X\right\} .
$$

The main property of the subdifferential mapping is the following proposition.

Proposition. Let $\varphi: X \rightarrow \mathbf{R} \cup\{\infty\}$ be proper convex weaklower semicontinuous. Then, the subdifferential mapping $\partial \varphi$ is minimal weak $^{*}$-cusco on $\operatorname{int} \operatorname{dom}(\varphi)$, and the set $A:=\{y \in Y: \partial \varphi(y) \cap X \neq \emptyset\}$ is dense in $\operatorname{dom}(\varphi)$.

3. WRNP and weakly single-valuedness of minimal weak*cusco. Let $X$ and $Y$ be Banach spaces and $f$ a real valued function on $Y$. For $A \subseteq Y$, the oscillation of $f$ on $A$ is the

$$
O(f, A)=\sup \{|f(y)-f(x)|: x, y \in Y\}
$$


and the oscillation of $f$ at a point $x \in Y$ is

$$
O(f, x)=\inf \{O(f, U): U \subseteq Y \text { is open and } x \in U\} .
$$

If $K$ is a nonempty bounded subset of $X^{*}$, then a weak ${ }^{*}$-open slice of $K$ is a set of the form

$$
S(K, x, \alpha)=\left\{x^{*} \in K:\left\langle x^{*}, x\right\rangle>\sup \langle x, K\rangle-\alpha\right\},
$$

where $x \in X, \alpha>0$.

Definition 3.1. $X^{*}$ is said to have the weak Radon-Nikodým property provided, for every nonempty weak*-compact convex $K$ of $X^{*}, \varepsilon>0$ and $x^{* *} \in X^{* *}$, there exists a weak*-open half-space $V$ in $X^{*}$ such that $K \cap V \neq \emptyset$ and $O\left(x^{* *}, K \cap V\right)<\varepsilon$, that is, there exists a weak*-open slice $S$ of $K$ such that $O\left(x^{* *}, S\right)<\varepsilon$. We say that a weak*-compact convex $K$ of $X^{*}$ has the weak Radon-Nikodým property if, for each weak*-compact convex $M$ of $K, \varepsilon>0$ and $x^{* *} \in X^{* *}$, there exists a weak*-open slice $S$ of $M$ such that $O\left(x^{* *}, S\right)<\varepsilon$.

Originally, Musial defined the vector integral version of the weak Radon-Nikodým property as mentioned in the introduction. That is, $X$ has the weak Radon-Nikodým property, if for each finite complete measure space $(\Omega, \Sigma, \mu)$ and each $\mu$-continuous $X$-valued countably additive vector measure $\nu: \Sigma \rightarrow X$ of bounded variation, there exists a Pettis integrable function $f: \Omega \rightarrow X$ such that $\nu(E)=P-\int_{E} f d \mu$ for all $E \in \Sigma$ (see [9]). Also, Riddle, Saab and Uhl defined that a nonempty subset $K$ of $X$ has the weak Radon-Nikodým property if, for each finite complete measure space $(\Omega, \Sigma, \mu)$ and each $\mu$-continuous $X$-valued countably additive vector measure $\nu: \Sigma \rightarrow X$ of bounded variation satisfying for all $E \in \Sigma$ the inclusion $\nu(E) \in \mu(E) \cdot K$, there exists a $K$-valued Pettis integrable function $f: \Omega \rightarrow K$ such that

$$
\nu(E)=P-\int_{E} f d \mu,
$$

for all $E \in \Sigma$ (see [1]). On the other hand, Saab and Saab proved that Riddle's definition of the weak Radon-Nikodým property for dual Banach space $X^{*}$ is equivalent to Definition 3.1. Furthermore, they showed that Riddle's definition of the weak Radon-Nikodým property for weak ${ }^{*}$-compact convex subsets of $X^{*}$ is equivalent to that 
of Definition 3.1 (see $[\mathbf{1 3}, \mathbf{1 4}, \mathbf{1 5}]$ ). In this paper, we use Definition 3.1 for the geometrical advantage.

Remark 3.2. Generally, if $X^{*}$ has the Radon-Nikodým property, then $X^{*}$ has the weak Radon-Nikodým property. However, the converse is not true. For example, $J T^{*}$ does not have the Radon-Nikodým property but has the weak Radon-Nikodým property where $J T$ is the James tree space (see [6]).

Definition 3.3. A set-valued mapping $T: Y \rightarrow 2^{X}$ is said to be weakly fragmented if, for every nonempty open set $U$ in $Y$ and $x^{*} \in X^{*}$ and any $\varepsilon>0$, there is a nonempty open set $U^{\prime} \subset U$ such that $O\left(x^{*}, T\left(U^{\prime}\right)\right)<\varepsilon$.

Clearly, if $T: Y \rightarrow 2^{X}$ is fragmented by norm, then $T$ is weakly fragmented. However, the converse is not true. After we prove a few results, we give this counterexample. The following lemma shows that nonempty-valued weakly fragmented mappings should be weakly singlevalued. It is derived from the analogous lemma of [7].

Lemma 3.4. Let $T: Y \rightarrow 2^{X}$ be a nonempty-valued weakly fragmented mapping and $x^{*} \in X^{*}$. Then, there exists a dense $G_{\delta}$ subset $D$ of $Y$ such that, at each point of $D, x^{*} T$ is single-valued and upper semicontinuous.

Proof. The essential idea of the proof comes from [11]. For each $k \in \mathbf{N}$, let

$$
V_{k}=\bigcup\left\{U: U \text { is an open subset of } Y \text { and } O\left(x^{*}, T(U)\right)<1 / k\right\} .
$$

Clearly, every $V_{k}$ is open. We claim that every $V_{k}$ is dense in $Y$. Indeed, let $U$ be a nonempty open subset of $Y$ and $k \in \mathbf{N}$. Since $T$ is a weakly fragmented mapping, there is a nonempty open set $U^{\prime} \subset U$ such that $O\left(x^{*}, T\left(U^{\prime}\right)\right)<1 / k$. Then we have $U^{\prime} \subset V_{k}$. This proves our claim. Now let $D=\bigcap_{k=1}^{\infty} V_{k}$. By the Baire category theorem, $D$ is a dense $G_{\delta}$ subset of $Y$. Take any $y \in D$ and take any sequence of open sets $\left(U_{k}\right)$ such that $y \in U_{k}$ and $O\left(x^{*}, T\left(U_{k}\right)\right)<1 / k$. Then we have 
$T(y) \subseteq \bigcap_{k=1}^{\infty} T\left(U_{k}\right)$. It follows that

$$
x^{*} T(y) \subseteq \bigcap_{k=1}^{\infty} x^{*} T\left(U_{k}\right) .
$$

Since $\lim _{k \rightarrow \infty} O\left(x^{*}, T\left(U_{k}\right)\right)=0, x^{*} T(y)$ is a singleton. Thus, there exists $a \in \mathbf{R}$ such that $\{a\}=x^{*} T(y)=\bigcap_{k=1}^{\infty} x^{*} T\left(U_{k}\right)$. For the second part, take any $\varepsilon>0$. Since $\{a\}=\bigcap_{k=1}^{\infty} x^{*} T\left(U_{k}\right)$, there exists a $k \in \mathbf{N}$ such that $x^{*} T\left(U_{k}\right) \subseteq B(a, \varepsilon)$ where $U_{k}$ is an open set containing $y$. Hence, $x^{*} T$ is upper semicontinuous on $D$.

Now we are going to reveal the relation between the weak RadonNikodým property and the weakly single-valuedness of minimal weak*cusco.

Proposition 3.5. Let $T: Y \rightarrow 2^{X^{*}}$ be minimal weak*-cusco and $x^{* *} \in X^{* *}$. If there exists a weak ${ }^{*}$-compact convex $A$ with the weak Radon-Nikodym property such that the set $\{y \in Y: T(y) \cap A \neq \emptyset\}$ is dense in $Y$, then there exists a dense $G_{\delta}$ subset $D$ of $Y$ such that, at each point of $D, x^{* *} T$ is single-valued and upper semicontinuous.

Proof. By Lemma 3.4, we have enough to show that $T$ is weakly fragmented. Let $U$ be any nonempty open set in $Y$. Define $S: Y \rightarrow 2^{X^{*}}$ by $S(y)=T(y) \cap A$. Since $\operatorname{dom}(S)$ is dense in $Y, \overline{S(U)} w^{*}$ is a nonempty weak*-compact convex subset of $A$. Since $A$ has the weak RadonNikodým property, there exists a weak*-open half-space $V$ in $X^{*}$ such that $\overline{S(U)} w^{*} \cap V \neq \emptyset$ and $O\left(x^{* *}, \overline{S(U)} w^{*} \cap V\right)<\varepsilon$. It is easy to see that $S(U) \cap V \neq \emptyset$. By the minimality property (i) for $T$, there exists a nonempty open set $U^{\prime} \subset U$ such that $T\left(U^{\prime}\right) \subset V$. Take any nonempty open set $W \subset U^{\prime}$. Since $S(W) \cap \overline{S(U)}^{w^{*}} \neq \emptyset$ and $\overline{S(U)} w^{*}$ is a weak* compact convex in $X^{*}$, by the minimality property (ii) for $T$, we have $T\left(U^{\prime}\right) \subset \overline{S(U)}^{w^{*}}$. Hence, $T\left(U^{\prime}\right) \subset \overline{S(U)} w^{*} \cap V$, so we obtain $O\left(x^{* *}, T\left(U^{\prime}\right)\right)<\varepsilon$.

Remark 3.6. When $Y$ is a nonempty open subset of a Banach space $Z$ in Proposition 3.5, it is also true because $Y$ is a Baire space. 
The following theorem illustrates the counterexample as mentioned in the discussion before Lemma 3.4. Recall that a Banach space $X$ is said to be an Asplund space, provided every continuous convex function defined on $X$ is Fréchet differentiable at each point of some dense subset of $X$ (that is, $G_{\delta}$ subset).

Theorem 3.7. Whenever $X^{*}$ has the weak Radon-Nikodým property but not the Radon-Nikodým property, there exists a set-valued operator $T: X \rightarrow 2^{X^{*}}$ which $T$ is weakly fragmented, but is not fragmented by norm.

Proof. Suppose that $X^{*}$ has the weak Radon-Nikodým property but not the Radon-Nikodým property; for example, $X$ is the James tree space. Hence, $X$ is not Asplund, so there exists a real-valued convex continuous function $f$ on $X$ where the set

$$
A=\{x \in X: f \text { is Fréchet differentiable at } x\}
$$

is not dense in $X$. Then there exists a nonempty open $U$ in $X$ such that $U \cap A=\emptyset$. Let $\partial f: X \rightarrow 2^{X^{*}}$ be the subdifferential of $f$. Since $f$ is continuous, $\partial f$ is a nonempty set-valued mapping. Suppose that $f$ is fragmented by norm. Then, as mentioned in the preliminaries, there exists a dense $G_{\delta}$ subset $D$ of $X$ such that, at each point of $D$, $\partial f$ is a single-valued mapping and upper semicontinuous. Since $D$ is a dense subset of $X$, there exists $x_{0} \in D \cap U$ such that $\partial f$ is upper semicontinuous at $x_{0}$. Since $\partial f\left(x_{0}\right)$ is a singleton, $\partial f$ is norm-to-norm continuous at $x_{0}$; hence, $f$ is Fréchet differentiable at $x_{0}$. It follows that $U \cap A \neq \emptyset$, so it is a contradiction.

Now we show that $\partial f$ is weakly fragmented. Let $U$ be a nonempty open subset of $X$ and $\varepsilon>0$ and $x^{* *} \in X^{* *}$. Since $\partial f$ is minimal weak*cusco, by a standard argument, we may assume that $\partial f(U)$ is bounded. By the Alaoglu theorem, $\overline{\operatorname{co} \partial f(U)} w^{*}$ is the weak*-compact convex subset of $X^{*}$. Since $X^{*}$ has the weak Radon-Nikodým property, there exists a weak* ${ }^{*}$-open half-space $V$ in $X^{*}$ such that $\overline{\operatorname{co} \partial f(U)} w^{*} \cap V \neq \emptyset$ and $O\left(x^{* *}, \overline{\operatorname{co} \partial f(U)}{ }^{w^{*}} \cap V\right)<\varepsilon$. Then we have $V \cap \partial f(U) \neq \emptyset$. Indeed, we choose $x^{*} \in V \cap \overline{\operatorname{co} \partial f(U)}{ }^{w^{*}}$. Since $V$ is a weak ${ }^{*}$-open neighborhood of $x^{*}$, there exists $y^{*} \in \operatorname{co} \partial f(U)$ such that $y^{*} \in V$ and $y^{*}=\sum_{i=1}^{n} \alpha_{i} x_{i}^{*}$ where each $x_{i}^{*}$ is in $\partial f(U)$ and $\sum_{i=1}^{n} \alpha_{i}$ is a convex combination. 
Suppose that every $x_{i}^{*}$ is not in $V$. Put $K=\overline{\operatorname{co} \partial f(U)}^{w^{*}}$ and

$$
V=V(x, \alpha)=\left\{x^{*} \in X^{*}:\left\langle x^{*}, x\right\rangle>\sup _{x^{*} \in K}\left\langle x^{*}, x\right\rangle-\alpha\right\} .
$$

Since every $x_{i}^{*}(x) \leq \sup _{x^{*} \in K}\left\langle x^{*}, x\right\rangle-\alpha$, we have

$$
\alpha_{i} x_{i}^{*} \leq \alpha_{i}\left(\sup _{x^{*} \in K}\left\langle x^{*}, x\right\rangle-\alpha\right)
$$

Then it follows that

$$
\sum_{i=1}^{n} \alpha_{i} x_{i}^{*}(x) \leq \sup _{x^{*} \in K}\left\langle x^{*}, x\right\rangle-\alpha ;
$$

hence, we obtain $y^{*}(x) \leq \sup _{x^{*} \in K}\left\langle x^{*}, x\right\rangle-\alpha$, so it is a contradiction. Thus, there exists one $x_{i}^{*}$ such that $x_{i}^{*} \in V$. Therefore, we have $V \cap \partial f(U) \neq \emptyset$. Then, by the minimality property (i) for $\partial f$, there exists a nonempty open set $U^{\prime} \subset U$ such that $\partial f\left(U^{\prime}\right) \subset V$. Clearly, we have $\partial f\left(U^{\prime}\right) \subset \overline{\operatorname{co} \partial f(U)} w^{*}$. Hence, $\partial f\left(U^{\prime}\right) \subset \overline{\operatorname{co} \partial f(U)} w^{*} \cap V$, so we obtain $O\left(x^{* *}, \partial f\left(U^{\prime}\right)\right)<\varepsilon$.

By the proof of Theorem 3.7, we obtain the following corollary.

Corollary 3.8. Let $T: Y \rightarrow 2^{X^{*}}$ be minimal weak*-cusco and $x^{* *} \in X^{* *}$. If $X^{*}$ has the weak Radon-Nikodym property, then there exists a dense $G_{\delta}$ subset $D$ of $Y$ such that, at each point of $D, x^{* *} T$ is single-valued and upper semicontinuous.

4. Main theorem. Let $K$ be a weak* compact convex subset of $X^{*}$ and $x \in X$. An extreme point $x^{*}$ of $K$ is weak* exposed by $x$ if, for $\left\{x_{n}^{*}\right\} \subset K$

$$
\left\langle x_{n}^{*}, x\right\rangle \longrightarrow\left\langle x^{*}, x\right\rangle=\sup _{y^{*} \in K}\left\langle y^{*}, x\right\rangle
$$

implies

$$
x_{n}^{*} \stackrel{w^{*}}{\longrightarrow} x^{*} .
$$

Let $x^{* *}$ be in $X^{* *}$. An extreme point $x^{*}$ of $K$ is $x^{* *}$-weak ${ }^{*}$ exposed by $x$ if, for $\left\{x_{n}^{*}\right\} \subset K$,

$$
\left\langle x_{n}^{*}, x\right\rangle \longrightarrow\left\langle x^{*}, x\right\rangle=\sup _{y^{*} \in K}\left\langle y^{*}, x\right\rangle
$$


implies

$$
x^{* *}\left(x_{n}^{*}\right) \longrightarrow x^{* *}\left(x^{*}\right) .
$$

Furthermore, an extreme point $x^{*}$ of $K$ is an $x^{* *}$-weak ${ }^{*}$ exposed point of $K$ if there exists one $x \in X$ which $x^{*}$ is $x^{* *}$-weak* exposed by $x$.

Remark 4.1. It is easy to see that $x^{*}$ is an $x^{* *}$-weak* exposed point of $K$ if and only if, for every $\varepsilon>0$, there exists a weak ${ }^{*}$-open slice $S(K, x, a)$ of $K$ with $O\left(x^{* *}, S\right)<\varepsilon$ (see [5]).

The following example shows that there exist $x^{*} \in X^{*}, x^{* *} \in X^{* *}$ and $x \in X$ such that $x^{*}$ is $x^{* *}$-weak* exposed by $x$ but $x^{*}$ is not weak* exposed by $x$.

Example 4.2. Let $X$ denote the Banach space $c_{0}$. Then $X^{*}=\ell_{1}$ and $X^{* *}=\ell_{\infty}$. Let $e_{n}$ be the unit vectors in $\ell_{1}$ and

$$
D_{1}=\left\{e_{n}: n \geq 3\right\} \cup\left\{e_{1}+e_{2}+e_{n}: n \geq 3\right\}
$$

and $K$ the weak*-closure of the convex hull of $D_{1}$. Then, we have that $0 \in K$ and is an extreme point of $K$. Let $x^{* *}=$ $(-1,1,0,0,0, \ldots)$. Then 0 is a $x^{* *}$-weak* exposed point of $K$. Indeed, consider $x=(-1,1,0,0,0, \ldots)$. Then, we have $\sup _{y^{*} \in K}\left\langle y^{*}, x\right\rangle=0$ because $(-1,1,0,0,0, \ldots)\left(y^{*}\right)=0$ for every $y^{*} \in D_{1}$. Since $x=x^{* *}$, we obtain that 0 is $x^{* *}$-weak ${ }^{*}$ exposed by $x$. However, 0 is not weak* exposed by $x$. To show this, we consider $\left(e_{1}+e_{2}+e_{n}\right)_{n \geq 3}$ in $K$. Since $\left(e_{1}+e_{2}+e_{n}\right)(x)=\left(e_{1}+e_{2}+e_{n}\right)(-1,1,0,0,0, \ldots)=0$, we have $\left(e_{1}+e_{2}+e_{n}\right)(x) \rightarrow\langle 0, x\rangle$, but $\left(e_{1}+e_{2}+e_{n}\right)\left(e_{1}\right) \nrightarrow 0$; hence, 0 is not weak* exposed by $x$.

Definition 4.3. For a proper lower semicontinuous $f: X^{*} \rightarrow \mathbf{R} \cup\{\infty\}$ and $y^{* *} \in X^{* *}$, the perturbed function $f-y^{* *}$ is said to attain a weak*minimum on $X^{*}$ if there exists $x_{0}^{*} \in X^{*}$ such that, for every sequence $\left\{x_{n}^{*}\right\} \subset X^{*}$,

$$
f\left(x_{n}^{*}\right)-\left\langle y^{* *}, x_{n}^{*}\right\rangle \longrightarrow \inf \left(f-y^{* *}\right)
$$


implies

$$
x_{n}^{*} \stackrel{w^{*}}{\longrightarrow} x_{0}^{*} .
$$

Necessarily, $x_{0}^{*}$ is the unique weak ${ }^{*}$-minimizer of $f-y^{* *}$. Let $x^{* *} \in X^{* *}$. The perturbed function $f-y^{* *}$ is said to attain a $x^{* *}$-minimum on $X^{*}$ if there exists $x_{0}^{*} \in X^{*}$ such that, for every sequence $\left\{x_{n}^{*}\right\} \subset X^{*}$,

$$
f\left(x_{n}^{*}\right)-\left\langle y^{* *}, x_{n}^{*}\right\rangle \longrightarrow \inf \left(f-y^{* *}\right)
$$

implies

$$
x^{* *}\left(x_{n}^{*}\right) \longrightarrow x^{* *}\left(x_{0}^{*}\right) .
$$

Recall that $f$ is said to be Gâteaux differentiable at $x_{0}$ if there exists a $x^{*}$ (denoted by $\nabla_{G} f\left(x_{0}\right)$ ) such that

$$
\nabla_{G} f\left(x_{0}\right)=\lim _{t \rightarrow 0^{+}} \frac{f\left(x_{0}+t x\right)-f\left(x_{0}\right)}{t}
$$

for each $x \in X$. It is well known that if $f$ is Fréchet differentiable at $x$, then $f$ is Gâteaux differentiable at $x$. However, the converse is not true (see, e.g., [11]).

The following proposition shows that the weak*-minimizer of the perturbed function is related to the Gâteaux differentiability.

Proposition 4.4. Let $f: X^{*} \rightarrow \mathbf{R} \cup\{\infty\}$ be proper lower semicontinuous and $x_{0} \in \operatorname{int} \operatorname{dom}\left(f^{*}\right)$. The following are equivalent.

(a) $\partial f^{*}$ is single-valued at $x_{0}$.

(b) $f^{*}$ is Gâteaux differentiable at $x_{0}$.

(c) $f-x_{0}$ attains a weak $k^{*}$ minimum on $X^{*}$.

Proof. (a) $\Leftrightarrow$ (b) is clear. And (b) $\Leftrightarrow$ (c) can be proved from the argument of [7, Proposition 4]. For completeness, we just provide the proof of the $(\mathrm{b}) \Rightarrow(\mathrm{c})$ case. Let $\left(x_{n}^{*}\right)$ be in $X^{*}$ such that $f\left(x_{n}^{*}\right)-\left\langle x_{0}, x_{n}^{*}\right\rangle \rightarrow \inf \left(f-x_{0}\right)=-f^{*}\left(x_{0}\right)$. Since $f^{*}$ is Gâteaux differentiable at $x_{0}$, there exists an $x^{*}=\nabla_{G} f^{*}\left(x_{0}\right)$ such that

$$
\nabla_{G} f^{*}\left(x_{0}\right)(x)=\lim _{t \rightarrow 0^{+}} \frac{f^{*}\left(x_{0}+t x\right)-f^{*}\left(x_{0}\right)}{t}
$$


for each $x \in X$. We claim that $\left(x_{n}^{*}\right)$ weak ${ }^{*}$-converges to $x^{*}$. Let $x$ be in $X$ and $\varepsilon>0$. Then there exists $\delta>0$ such that if $0<t<\delta$, then

$$
0 \leq f^{*}\left(x_{0}+t x\right)-f^{*}\left(x_{0}\right)-\left\langle x^{*}, t x\right\rangle \leq \varepsilon t .
$$

For each $n \in \mathbf{N}$, we choose real $t_{n}$ such that $\left|\left\langle x_{n}^{*}-x^{*}, x\right\rangle\right| \leq\left\langle x_{n}^{*}-\right.$ $\left.x^{*}, t_{n} x\right\rangle+\varepsilon$. Indeed, since $x_{n}^{*}-x^{*}$ is continuous on $X$, we can choose real $r_{n}$ with $\left|r_{n}\right| \leq 1$ such that

$$
\left|\left\langle x_{n}^{*}-x^{*}, x\right\rangle\right| \leq\left|\left\langle x_{n}^{*}-x^{*}, r_{n} x\right\rangle\right|+\varepsilon .
$$

We put $t_{n}=r_{n}$ if $\left\langle x_{n}^{*}-x^{*}, r_{n} x\right\rangle \geq 0,-r_{n}$ otherwise. Then we have $\left|\left\langle x_{n}^{*}-x^{*}, x\right\rangle\right| \leq\left\langle x_{n}^{*}-x^{*}, t_{n} x\right\rangle+\varepsilon$. Now put $s_{n}=\delta t_{n}$ for each $n \in \mathbf{N}$. By the definition of $f^{*}$, we have

$$
\left\langle x_{n}^{*}, x_{0}+s_{n} x\right\rangle-f\left(x_{n}^{*}\right) \leq f^{*}\left(x_{0}+s_{n} x\right)
$$

for each $n \in \mathbf{N}$. Since $f\left(x_{n}^{*}\right)-\left\langle x_{0}, x_{n}^{*}\right\rangle \rightarrow-f^{*}\left(x_{0}\right)$, we obtain $f\left(x_{n}^{*}\right)-\left\langle x_{0}, x_{n}^{*}\right\rangle \leq-f^{*}\left(x_{0}\right)+\delta \varepsilon$ for large $n$. Hence, for large $n$, we have

$$
\begin{aligned}
\delta\left|\left\langle x_{n}^{*}-x^{*}, x\right\rangle\right| \leq & \left\langle x_{n}^{*}-x^{*}, \delta t_{n} x\right\rangle+\delta \varepsilon \\
= & \left\langle x_{n}^{*}, x_{0}+s_{n}\right\rangle-f\left(x_{n}^{*}\right) \\
& +f\left(x_{n}^{*}\right)-\left\langle x_{n}^{*}, x_{0}\right\rangle-\left\langle x^{*}, s_{n} x\right\rangle+\delta \varepsilon \\
\leq & f^{*}\left(x_{0}+s_{n} x\right)-f^{*}\left(x_{0}\right)+\delta \varepsilon-\left\langle x^{*}, s_{n} x\right\rangle+\delta \varepsilon \\
\leq & 3 \delta \varepsilon .
\end{aligned}
$$

This proves our claim.

Let $K$ be a nonempty convex subset of $X$ and $x^{* *} \in X^{* *}$. Then the indicator function $\delta_{K}$, denoted by $\delta_{K}(x)=0$ if $x \in K,=\infty$ otherwise, is a proper convex function which is lower semicontinuous if and only if $K$ is closed (see, e.g., [11]). The relationship between the $x^{* *}$-weak exposed point (the weak*-exposed point) and the $x^{* *}$-minimum (the weak*-minimum) is exhibited by the following lemma. The proof is trivial.

Lemma 4.5. Let $K$ be a weak* compact convex in $X^{*}$, and let $x^{* *}$ be in $X^{*}$. Then $x^{*} \in K$ is an $x^{* *}$-weak ${ }^{*}$ exposed point of $K$ with $x^{* *}$-weak exposing functional $x \in X$ if and only if $\delta_{K}-x$ attains a $x^{* *}$-minimum on $X^{*}$ at $x^{*}$. Furthermore, $x^{*} \in K$ is a weak ${ }^{*}$ exposed point of $K$ 
with weak* exposing functional $x \in X$ if and only if $\delta_{K}-x$ attains a weak*-minimum on $X^{*}$ at $x^{*}$.

Now we are going to prove our main theorem. To obtain this result, we need the well-known following theorem (see [2]). Note that, for any $\varepsilon>0$, the $\varepsilon$-subdifferential $\partial_{\varepsilon}$ is denoted by

$$
\partial f_{\varepsilon}(x)=\left\{x^{*} \in X^{*}:\left\langle x^{*}, y\right\rangle \leq f(x+y)-f(x)+\varepsilon, \text { for all } y \in X\right\} .
$$

Theorem 4.6 (Brøndsted-Rockafellar). Suppose that $f$ is a convex proper lower semicontinuous function on $X$. Then, given any point $x_{0} \in \operatorname{dom}(f), \varepsilon>0, \lambda>0$ and $x_{0}^{*} \in \partial_{\varepsilon} f\left(x_{0}\right)$, there exist $x \in \operatorname{dom}(f)$ and $x^{*} \in X^{*}$ such that

$$
x^{*} \in \partial f(x), \quad\left\|x-x_{0}\right\| \leq \varepsilon / \lambda, \quad \text { and } \quad\left\|x^{*}-x_{0}^{*}\right\| \leq \lambda .
$$

Theorem 4.7. Let $X$ and $Y$ be Banach spaces, and let $K$ be a nonempty weak* compact convex subset of $X^{*}$. The following are equivalent.

(a) For every minimal weak $k^{*}$-cusco $T: Y \rightarrow 2^{X^{*}}$ with $\{y \in Y: T(y) \cap$ $K \neq \emptyset\}$ dense in $Y$ and $x^{* *} \in X^{* *}$, there exists a dense $G_{\delta}$ subset $D$ of $Y$ such that $x^{* *} T$ is single-valued and upper semicontinuous at each point of $D$.

(b) For every proper convex weak-lower semicontinuous $f: X \rightarrow$ $\mathbf{R} \cup\{\infty\}$ with $\operatorname{dom}\left(f^{*}\right) \subset K$ and $x^{* *} \in X^{* *}$, there exists a dense $G_{\delta}$ subset $D$ of int dom $(f)$ such that $x^{* *} \partial f$ is single-valued and upper semicontinuous at each point of $D$.

(c) For every proper lower semicontinuous $f: X^{*} \rightarrow \mathbf{R} \cup\{\infty\}$ with $\operatorname{dom}(f) \subset K$ and $x^{* *} \in X^{* *}$, there exists a dense $G_{\delta}$ subset $D$ of int dom $\left(f^{*}\right)$ such that for each $x \in D, f-x$ attains a $x^{* *}$ minimum.

(d) Every nonempty weak* compact convex subset of $K$ has an $x^{* *}$ weak* ${ }^{*}$ exposed point of $K$.

(e) $K$ has the weak Radon-Nikodym property.

Proof. (d) $\Rightarrow$ (e) and (e) $\Rightarrow$ (a) are clear by Proposition 3.5 and Remark 4.1.

$(\mathrm{a}) \Rightarrow(\mathrm{b})$. We may assume that int $\operatorname{dom}(f) \neq \emptyset$. Observe that $\{x \in$ $\left.X: \partial f(x) \cap X^{*} \neq \emptyset\right\}=\{x \in X: \partial f(x) \cap K \neq \emptyset\}$. Since $f$ is a proper 
convex weak-lower semicontinuous, it follows from the preliminaries that $\partial f$ is minimal weak ${ }^{*}$-cusco and $\{x \in X: \partial f(x) \cap K \neq \emptyset\}$ is dense in $X$. Then, by (a), the conclusion follows.

(c) $\Rightarrow$ (d). Let $C$ be a nonempty weak* compact convex subset of $K$ and $x^{* *}$ in $X^{* *}$. Consider $\delta_{C}$. Then $\delta_{C}: X^{*} \rightarrow \mathbf{R} \cup\{\infty\}$ is proper lower semicontinuous with $\operatorname{dom}\left(\delta_{C}\right)=C \subset K$ and $\operatorname{dom}\left(\delta_{C}^{*}\right)=X$. By the assumption, there exists one $x \in X$ such that $\delta_{C}-x$ attains a $x^{* *}$-minimum. By Lemma 4.4, there exists a $x^{* *}$-weak ${ }^{*}$ exposed point of $C$.

(b) $\Rightarrow$ (c). Let $f: X^{*} \rightarrow \mathbf{R} \cup\{\infty\}$ be proper lower semicontinuous with $\operatorname{dom}(f) \subset K$ and $x^{* *} \in X^{* *}$. We may assume that int $\operatorname{dom}\left(f^{*}\right)$ is nonempty and $x^{* *} \in B_{X^{* *}}$. Since $f^{*}: X \rightarrow \mathbf{R} \cup\{\infty\}$ is proper weak-lower semicontinuous and $\operatorname{dom}\left(f^{* *}\right) \subset \overline{\operatorname{codom}}(f) \subset K$, by (b), there exists a dense $G_{\delta}$ subset $D$ of int $\operatorname{dom}\left(f^{*}\right)$ such that $x^{* *} \partial f^{*}$ is single-valued and upper semicontinuous at each point of $D$. Let $x \in D$. Now it is enough to show that $f-x$ attains the $x^{* *}$-minimum. That is, we prove that there exists an $x^{*} \in X^{*}$ such that, for every sequence $\left\{x_{n}^{*}\right\} \subset X^{*}$,

$$
f\left(x_{n}^{*}\right)-\left\langle x, x_{n}^{*}\right\rangle \longrightarrow \inf (f-x)
$$

implies

$$
x^{* *}\left(x_{n}^{*}\right) \longrightarrow x^{* *}\left(x^{*}\right) .
$$

Let $\varepsilon>0$ be given. Consider a sequence $\left\{x_{n}^{*}\right\} \subset X^{*}$ such that $f\left(x_{n}^{*}\right)-\left\langle x, x_{n}^{*}\right\rangle \rightarrow \inf (f-x)$. Then we have $f\left(x_{n}^{*}\right)-\left\langle x, x_{n}^{*}\right\rangle \rightarrow-f^{*}(x)$. Since $x^{* *} \partial f^{*}$ is upper semicontinuous at $x$, there exists $\delta>0$ such that, if $\|x-y\|<\delta$, then $x^{* *} \partial f^{*}(y) \subset B(a, \varepsilon)$ where $a=x^{* *} \partial f^{*}(x)$. Then there exists an $N \in \mathbf{N}$ such that, if $n>N$, then

$$
f\left(x_{n}^{*}\right)-\left\langle x, x_{n}^{*}\right\rangle \leq-f^{*}(x)+\varepsilon \delta .
$$

Fix any $n>N$. We claim that $x_{n}^{*} \in \partial_{\varepsilon \delta} f^{*}(x)$. Indeed, let $z$ be in $X$. Since $f^{*}(y)=\sup \left\{\left\langle y^{*}, y\right\rangle-f\left(y^{*}\right): y^{*} \in X^{*}\right\}$ for every $y \in X$, we have $\left\langle x_{n}^{*}, x+z\right\rangle-f\left(x_{n}^{*}\right) \leq f^{*}(x+z)$. Hence, we obtain

$$
\begin{aligned}
\left\langle x_{n}^{*}, z\right\rangle & =\left\langle x_{n}^{*}, x+z\right\rangle-\left\langle x_{n}^{*}, x\right\rangle \\
& \leq f^{*}(x+z)+f\left(x_{n}^{*}\right)-f\left(x_{n}^{*}\right)-f^{*}(x)+\varepsilon \delta \\
& =f^{*}(x+z)-f^{*}(x)+\varepsilon \delta .
\end{aligned}
$$


This proves our claim. Now we consider $x^{*} \in \partial f^{*}(x)$. Then we are going to prove that $\left|x^{* *}\left(x_{n}^{*}\right)-x^{* *}\left(x^{*}\right)\right|<2 \varepsilon$. Since $f^{*}$ is proper convex lower semicontinuous on $X$ and $x_{n}^{*} \in \partial_{\varepsilon \delta} f^{*}(x)$ and $x \in \operatorname{dom}\left(f^{*}\right)$, by Brøndsted-Rockafellar theorem, there exist $y \in \operatorname{dom}\left(f^{*}\right)$ and $\left(x^{n}\right)^{*} \in$ $X^{*}$ such that $\left(x^{n}\right)^{*} \in \partial f^{*}(y),\|x-y\| \leq \varepsilon \delta / \varepsilon$, and $\left\|x_{n}^{*}-\left(x^{n}\right)^{*}\right\| \leq \varepsilon$. Then, we have $\|x-y\|<\delta$ and $\left|x^{* *}\left(x_{n}^{*}-\left(x^{n}\right)^{*}\right)\right| \leq \varepsilon$. Since $\left(x^{n}\right)^{*} \in \partial f^{*}(y)$ and $x^{*} \in \partial f^{*}(x)$, we obtain

$$
\left|x^{* *}\left(x^{*}\right)-x^{* *}\left(x^{n}\right)^{*}\right|=\left|a-x^{* *}\left(x^{n}\right)^{*}\right|<\varepsilon .
$$

Hence, we have

$$
\left|x^{* *}\left(x_{n}^{*}-x^{*}\right)\right| \leq\left|x^{* *}\left(x_{n}^{*}-\left(x^{n}\right)^{*}\right)\right|+\left|x^{* *}\left(\left(x^{n}\right)^{*}-x^{*}\right)\right| \leq 2 \varepsilon .
$$

This completes the proof.

In the proof of $(b) \Rightarrow(c)$, we showed the following fact that we state as a proposition.

Proposition 4.8. Let $K$ be a nonempty weak* compact convex subset of $X^{*}$ and $f: X^{*} \rightarrow \mathbf{R} \cup\{\infty\}$ proper lower semicontinuous with $\operatorname{dom}(f) \subset K$ and $x^{* *} \in X^{* *}$. Suppose that there exists a dense $G_{\delta}$ subset $D$ of int dom $\left(f^{*}\right)$ such that $x^{* *} \partial f^{*}$ is single-valued and upper semicontinuous at each point of $D$. Then, for each $x \in D$, every $x^{*} \in \partial f^{*}(x)$ is an $x^{* *}$-minimizer of $f-x$.

Finally, we provide equivalent conditions for the weak RadonNikodým property of a dual Banach space $X^{*}$. The proof is similar to that of Theorem 4.6, and we use Remark 4.1 and Corollary 3.8.

Corollary 4.9. Let $X$ and $Y$ be Banach spaces. The following are equivalent.

(a) For every minimal weak $k^{*}$ cusco $T: Y \rightarrow 2^{X^{*}}$ and $x^{* *} \in X^{* *}$, there exists a dense $G_{\delta}$ subset $D$ of $Y$ such that $x^{* *} T$ is single-valued and upper semicontinuous at each point of $D$.

(b) For every proper convex weak-lower semicontinuous $f: X \rightarrow \mathbf{R} \cup$ $\{\infty\}$ and $x^{* *} \in X^{* *}$, there exists a dense $G_{\delta}$ subset $D$ of $\operatorname{int} \operatorname{dom}(f)$ such that $x^{* *} \partial f$ is single-valued and upper semicontinuous at each point of $D$. 
(c) For every proper lower semicontinuous $f: X^{*} \rightarrow \mathbf{R} \cup\{\infty\}$ and $x^{* *} \in X^{* *}$, there exists a dense $G_{\delta}$ subset $D$ of int dom $\left(f^{*}\right)$ such that, for each $x \in D, f-x$ attains a $x^{* *}$-minimum.

(d) Every nonempty weak ${ }^{*}$ compact convex subset of $X^{*}$ has a $x^{* *}$ weak* exposed point.

(e) $X^{*}$ has the weak Radon-Nikodým property.

\section{REFERENCES}

1. R.D. Bourgin, Geometric aspects of convex sets with the Radon-Nikodým property, Lect. Notes in Math., 993, Springer, Berlin, 1983.

2. A. Brøndsted and R.T. Rockafellar, On the subdifferentiability of convex functions, Proc. Amer. Math. Soc. 16 (1965), 605-611.

3. J. Diestel, and J.J. Uhl, Jr., Vector measures, American Mathematical Society Surveys 15, Providence, Rhode Island, 1977.

4. L. Drewnowski and I. Labuda, On minimal convex usco and maximal monotone maps, Real Anal. Exch. 15 (1989/90), 729-742.

5. V. Farmaki, A geometric characterization of the weak Radon-Nikodým property in dual Banach spaces, Rocky Mountain J. Math. 25 (1995), 611-617.

6. R.C. James, A separable somewhat reflexive Banach spaces with nonseparable dual, Bulletin Amer. Math. Soc. 80 (1974), 738-743.

7. M. Lassonde, Aspund spaces, Stegall variational principle and the RNP, SetValued Anal. 17 (2009), 183-193.

8. J. Lindenstrauss and C. Stegall, Examples of separable spaces which do not contain $\ell_{1}$ and whose duals are non-separable, Stud. Math. 54 (1975), 81-105.

9. K. Musial, The weak Radon-Nikodým property in Banach space, Stud. Math. 64 (1978), 151-174.

10. A. Pelczynski, On Banach spaces containing $L_{1}$, Stud. Math. 30 (1968), $231-246$.

11. R.R. Phelps Convex functions, monotone operators and differentiability, Lect. Notes Math. 1364 (1993), Springer, Berlin.

12. H.P. Rosenthal, Characterization of Banach spaces containing $\ell_{1}$, Proc. Nar. Acad. Sci. 71 (1974), 2411-2413.

13. E. Saab and P. Saab, Some characterizations of weak Radon-Nikodým sets, Proc. Amer. Math. Soc. 86 (1982), 307-311.

14. , A dual geometric characterization of Banach spaces not containing $\ell_{1}$, Pac. J. Math. 105 (1983), 415-425.

15. Sets with the weak Radon-Nikodým property in dual Banach spaces, Ind. Univ. Math. J. 32 (1983), 527-541.

Institute for Ubiquitous Information Technology and Applications, Konkuk University, Seoul, 143-701, Korea

Email address: northstar@kaist.ac.kr 